\title{
A Perturbation Finite Element Method for Modeling Moving Conductive and Magnetic Regions without Remeshing
}

\author{
Patrick Dular and Ruth V. Sabariego \\ University of Liège, Dept. of Electrical Engineering and Computer Science, B28, B-4000 Liège, Belgium \\ E-mail: Patrick.Dular@ulg.ac.be
}

\begin{abstract}
Purpose - To develop a sub-domain perturbation technique for efficiently modeling moving systems in magnetodynamics with a magnetic field $h$-conform finite element formulation.

Design/methodology/approach - A reference problem is first solved in a global mesh excluding some moving regions and thus avoiding the inclusion of their meshes. Its solution gives the sources for a sequence of perturbation problems with the supplementary moving magnetic and conductive regions. Each of these sub-problems requires an appropriate proper volume mesh of the associated moving region and its surrounding region, with no need of interconnection. The solutions are transferred from one problem to the other through projections of source fields between meshes.

Findings - The consideration of sub-problems and associated sources, in a sequence of perturbation problems, leads to a significant speed-up of the repetitive solutions in analyses of moving systems. A free movement in any direction can be considered with no need of remeshing.

Originality/value - When working with the perturbation fields, the volume sources can be limited to the moving regions, what allows for homogeneous perturbation boundary conditions and reduces the computational efforts for projecting and evaluating the sources. The curl-conformity of the unknown magnetic field is preserved during the whole process thanks to the use of edge finite elements for both the magnetic field and the intermediate source quantities. The sub-problem approach also gives an easy way to directly express the time derivatives in moving frames.
\end{abstract}

Keywords - Eddy currents, finite element method, moving systems, perturbation method

Paper type - Research paper

\section{INTRODUCTION}

Finite element (FE) analyses of moving systems usually require repetitive computations and remeshing of some regions. This way, a new complete FE solution for any new position of the moving regions is carried out.

An unperturbed solution followed by perturbed solutions with additional magnetic and conductive regions has been shown to be of interest for computing the ensuing field distortions and induced effects (Badics, Z. et al., 1997; Sabariego and Dular, 2006; Dular and Sabariego, 2007). Benefits are particularly aimed for allowing different problem-adapted meshes and for computational efficiency due to the reduced size of each sub-problem. Such a perturbation technique is here developed for the analysis of moving systems.

A perturbation method for solving magnetodynamic problems with moving parts in two separate steps is developed for a magnetic field $\boldsymbol{h}$-conform $\mathrm{FE}$

This work was partly supported by the Belgian Science Policy (IAP P6/21) and the Belgian French Community (Research Concerted Action ARC 03/08-298). P. Dular is also with the Belgian National Fund for Scientific Research (F.N.R.S.). formulation. It is an extension of the method proposed by the authors in (Dular and Sabariego, 2007) where only fixed regions are considered. A reference problem is first solved in a global domain excluding additional moving magnetic and conductive regions and thus avoiding the inclusion of their meshes. Its solution gives then the sources for the added moving regions in so-called perturbation problems, each one defined for each new position of these regions.

The advantages of defining volume sources limited to the moving regions, for feeding the perturbation problems, will be pointed out. Application examples will illustrate and validate the method, highlighting the parameters that affect the accuracy of the solution and the computational efficiency. The numerical tools proper to the perturbation technique will thus appear adapted to a wide range of magnetodynamic problems, with induced effects due to varying fields in either fixed or moving systems, in both frequency and time domains.

\section{Moving Systems Defining a Sequence of Perturbation Problems - THE STRONG FORMULATIONS}

\section{A. Unperturbed and Perturbed Problems}

Maxwell equations are to be solved in a domain $\Omega$, with boundary $\partial \Omega$ (possibly at infinity), of the $2-\mathrm{D}$ or $3-\mathrm{D}$ Euclidean space. The eddy current conducting part of $\Omega$ is denoted $\Omega_{c}$ and the non-conducting one $\Omega_{c}{ }^{C}$, with $\Omega=\Omega_{c} \cup \Omega_{c}{ }^{C}$. Massive conductors belong to $\Omega_{c}$.

The equations and relations governing the magnetodynamic (eddy current) problem in $\Omega$ are

$$
\begin{gathered}
\operatorname{curl} \boldsymbol{h}=\boldsymbol{j}, \operatorname{curl} \boldsymbol{e}=-\partial_{\mathrm{t}} \boldsymbol{b}, \operatorname{div} \boldsymbol{b}=0, \\
\boldsymbol{b}=\mu \boldsymbol{h}, \boldsymbol{j}=\sigma \boldsymbol{e},
\end{gathered}
$$

where $\boldsymbol{h}$ is the magnetic field, $\boldsymbol{b}$ is the magnetic flux density, $\boldsymbol{e}$ is the electric field, $\boldsymbol{j}$ is the electric current density (including source and eddy currents), $\mu$ is the magnetic permeability and $\sigma$ is the electric conductivity. Appropriate boundary conditions are to be defined as well.

A so-called unperturbed problem is first defined in $\Omega$ with the absence of a moving conductive region $\Omega_{c, p} \subset \Omega_{c}$ (which, at the discrete level, is not even described in the mesh of $\Omega$ ) (Fig. 1, left). Its solution constitutes a source for the moving region, i.e. the socalled perturbing region. For each position of the moving region, a perturbed problem is solved in a domain $\Omega_{p}$ including $\Omega_{c, p}$ and its neighborhood, adequately defined 
and meshed (Fig. 1, middle and right). The moving region can be magnetic but non-conductive, which easily particularizes the developed method.

Hereafter, the subscripts $u$ and $p$ will refer to unperturbed and perturbed quantities, respectively. The eddy currents induced by the movement, as well as the associated field distortion, will be the perturbed quantities of interest.
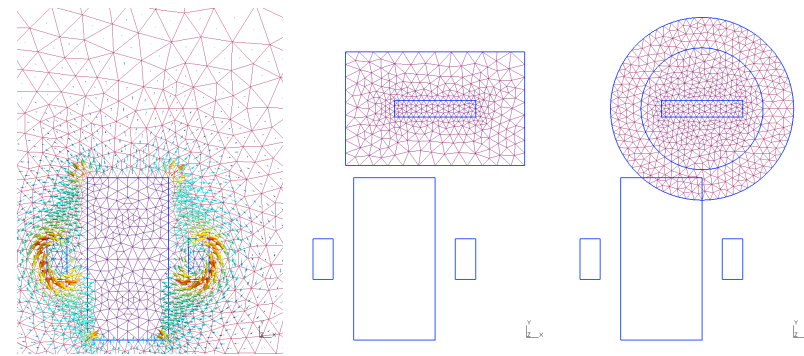

Fig. 1. Meshes of the unperturbed problem (left; with field $\boldsymbol{h}_{u}$ ) and perturbation problems with finite (middle) and infinite (right; (Imhoff et $a l ., 1990)$ ) boundaries. Any intersection of perturbation boundaries (or $\Omega_{p} \backslash \Omega_{c, p}$ ) with the unperturbed problem material regions is allowed.

\section{B. Perturbation Problem}

Particularizing (1) and (2) for both the unperturbed and perturbed quantities, and subtracting the unperturbed equations from the perturbed ones, a perturbation problem is defined in $\Omega_{p}$ (initially in $\Omega$ ) (Badics, Z. et al., 1997; Sabariego and Dular, 2006; Dular and Sabariego, 2007). Expressing the resulting equations in terms of the field distortions $\boldsymbol{h}=\boldsymbol{h}_{p}-\boldsymbol{h}_{u}$ and $\boldsymbol{e}=\boldsymbol{e}_{p}-\boldsymbol{e}_{u}$, one gets

$$
\begin{gathered}
\operatorname{curl} \boldsymbol{h}=\boldsymbol{j}, \operatorname{curl} \boldsymbol{e}=-\partial_{\mathrm{t}} \boldsymbol{b}, \\
\boldsymbol{b}=\mu_{p} \boldsymbol{h}+\boldsymbol{b}_{s}, \boldsymbol{j}=\sigma_{p} \boldsymbol{e}+\boldsymbol{j}_{s},
\end{gathered}
$$

where the so-defined volume sources $\boldsymbol{b}_{S}$ and $\boldsymbol{j}_{s}$ are obtained from the unperturbed solution as

$$
\begin{aligned}
& \boldsymbol{j}_{s}=\left(\sigma_{p}-\sigma_{u}\right) \boldsymbol{e}_{u} \text { in } \Omega_{c, p}, \\
& \boldsymbol{b}_{s}=\left(\mu_{p}-\mu_{u}\right) \boldsymbol{h}_{u} \text { in } \Omega_{c, p} .
\end{aligned}
$$

The boundary conditions to be considered are

$$
\boldsymbol{n} \times\left.\boldsymbol{h}\right|_{\partial \Omega_{p}}=0 \text { or } \boldsymbol{n} \times\left.\boldsymbol{e}\right|_{\partial \Omega_{p}}=0
$$

\section{Studied domain and boundary conditions of the perturbation problem}

The perturbation problem (3)-(7) is actually rigorously defined in the whole studied domain $\Omega$ taking account of the geometrical and material characteristics of the initial unperturbed problem. For the sake of convenience, some of the initial details of the unperturbed problem are disregarded. The so-modified studied domain $\Omega_{p}$ can be a portion of $\Omega$ or not, with or without considering initial materials, these being also possibly simplified (Dular and Sabariego, 2007).

For a bounded domain $\Omega_{p}$, the conditions ( $\left.7 \mathrm{a}-\mathrm{b}\right)$ neglect the distortion at a certain distance from $\Omega_{c, p}$, which is actually only correct at infinity (for $\Omega_{p}$ extended to the whole space). The homogeneous nature of the boundary conditions $(7 a-b)$ is preserved even with the approximation of a bounded domain $\Omega_{p}$. The perturbed problem, if solved for the unknown fields $\boldsymbol{h}_{p}$ and $\boldsymbol{e}_{p}$, would imply non-homogeneous boundary conditions depending on the location on $\partial \Omega_{p}$. The unperturbed fields would thus serve as surface sources, to be projected on the perturbed mesh boundary $\partial \Omega_{p}$. However, such conditions can only be applied if a finite boundary $\partial \Omega_{p}$ is defined. Indeed an infinite boundary would support a zero source, with no information at all for the perturbed problem. The unperturbed field $\boldsymbol{h}_{u}$ could alternatively be used as a classical volume source magnetic field in the whole $\Omega_{p}$, but with the disadvantage of needing its projection and evaluation on the whole domain.

At the discrete level, neglecting some material or geometrical details in some sub-problems enables the meshes of both unperturbed and perturbed problems to be significantly simplified. Each problem asks for mesh refinement of different regions, with no need of interconnection. In other words, each mesh can be generated independently of the other with the only requirement of sufficient fineness to accurately approximate its inherent field components.

For close relative positions of the source and perturbing regions, or for strong interactions between them, a more accurate solution can be obtained via an iterative procedure that calculates successive perturbations from one region to the other. Each subproblem is responsible for giving the suitable correction, as a perturbation, for the details it considers, with the accuracy limited by the fineness of its mesh.

\section{Sources of the perturbation problem}

The sources $\boldsymbol{j}_{S}$ (5) and $\boldsymbol{b}_{S}$ (6) only act in the regions where a change of conductivity or permeability occurs, i.e. in $\Omega_{c, p}$. This is a consequence of directly using the distortions $\boldsymbol{h}$ and $\boldsymbol{e}$ as unknowns instead of the perturbed fields $\boldsymbol{h}_{p}$ and $\boldsymbol{e}_{p}$. This property, together with the homogeneous nature of the boundary conditions for any location of $\partial \Omega_{p}$, provides computational advantages.

The source $\boldsymbol{b}_{s}$ (6) can be directly obtained from the known field $\boldsymbol{h}_{u}$. As for the source current density $\boldsymbol{j}_{s}(5)$, it is to be obtained from the still undetermined unperturbed electric field $\boldsymbol{e}_{u}$. Indeed, in the considered case, with $\sigma_{u}=0$ and $\sigma_{p} \neq 0$ in $\Omega_{c, p}$, the field $\boldsymbol{e}_{u}$ is unknown in any non-conducting regions. This is an additional difficulty in comparison with the complementary case $\sigma_{u} \neq 0$ and $\sigma_{p}=0$ studied in (Sabariego and Dular, 2006). The determination of $\boldsymbol{e}_{u}$ requires solving an electric problem defined by the Faraday and electric conservation equations, together with the electric constitutive relation.

\section{E. Unperturbed electric field as intermediary source}

The unperturbed electric field $\boldsymbol{e}_{u}$ is to be determined in the added moving conductive region $\Omega_{c, p}$, as an 
intermediary source for $\boldsymbol{j}_{S}(5)$. It has to be calculated via an electric problem defined only in $\Omega_{c, p}$ by

$$
\operatorname{curl} \boldsymbol{e}_{u}=-\partial_{\mathrm{t}}\left(\mu_{u} \boldsymbol{h}_{u}\right), \operatorname{div} \boldsymbol{d}_{u}=0, \boldsymbol{d}_{u}=\varepsilon_{u} \boldsymbol{e}_{u},
$$

where the just defined quantities are the unperturbed electric flux density $\boldsymbol{d}_{u}$ and the electric permittivity $\varepsilon_{u}$. Boundary conditions have to be defined for the trace $\boldsymbol{n} \times \boldsymbol{e}_{u}$ on $\partial \Omega_{c, p}$. Equation (8b) assumes that no charge density exists in $\Omega_{c, p}$.

\section{Magnetic Field CONFORM WeAK FORMULATIONS}

\section{A. The unperturbed solution as a source}

The conformity of $\boldsymbol{h}$ (conservation of its circulation or currents) can be assured through the definition of a magnetic scalar potential $\phi$ in $\Omega_{c}{ }^{C}$, with $\boldsymbol{h}=-\operatorname{grad} \phi$; this potential is discretized with nodal FEs. A total potential is multivalued when $\Omega_{c}{ }^{C}$ is multiply connected, case in which surface cuts must be defined to ensure a single valued potential. However a reduced potential can be continuously defined in the whole $\Omega_{c}{ }^{C}$ by adding source fields associated to each multiply connected portion of $\Omega_{c}$ (Dular et al., 2000). In $\Omega_{c}$, the conformity of $\boldsymbol{h}$ is assured by properly defining its function space, through edge FEs at the discrete level to strongly express (1a).

The unperturbed field distribution is first calculated in $\Omega$ as the solution of an eddy current problem with a magnetic field conform FE formulation, obtained from the weak form of the Faraday equation (1b), i.e. (Dular et al., 2000),

$$
\begin{aligned}
& \left(\partial_{t}\left(\mu_{u} \boldsymbol{h}_{u}\right), \boldsymbol{h}^{\prime}\right)_{\Omega^{+}}+\left(\sigma_{u}^{-1} \operatorname{curl} \boldsymbol{h}_{u}, \operatorname{curl} \boldsymbol{h}^{\prime}\right)_{\Omega_{c} \backslash \Omega_{c, p}} \\
& +\left(\boldsymbol{e}_{u}, \operatorname{curl} \boldsymbol{h}^{\prime}\right)_{\Omega_{c, p}}+<\boldsymbol{n} \times \boldsymbol{e}_{u}, \boldsymbol{h}^{\prime}>_{\Gamma_{e}}=0, \forall \boldsymbol{h}^{\prime} \in F^{1}(\Omega),
\end{aligned}
$$

where $F^{1}(\Omega)$ is the curl-conform function space defined on $\Omega$ and containing the basis functions for $\boldsymbol{h}_{u}$ as well as for the test function $\boldsymbol{h}^{\prime} ;(\cdot, \cdot)_{\Omega}$ and $<\cdot, \cdot>_{\Gamma}$ respectively denote a volume integral in $\Omega$ and a surface integral on $\Gamma$ of the product of their vector field arguments. The surface term in (9) accounts for the natural boundary or interface conditions.

The volume integral term in $\Omega_{c, p}$ in (9), involving the electric field $\boldsymbol{e}_{u}$, is null at this step (test function $\boldsymbol{h}^{\prime}$ is curl-free because $\Omega_{c, p}$ is not yet subject to eddy currents). As a consequence, $\boldsymbol{e}_{u}$ in any portion of $\Omega_{c}{ }^{C}$ cannot be part of a single magnetodynamic solution. Its contribution in (9) will be further used by a perturbation problem (with non-curl-free $\boldsymbol{h}^{\prime}$ ). As already introduced, parts of the unperturbed solution will serve as sources for the perturbation problem.

\section{B. Sequence of perturbation eddy current problems}

For each added moving domain $\Omega_{c, p}$, the magnetic field conform FE formulation of the perturbation problem (3)-(7) is obtained as the weak form of the perturbation Faraday equation (3b), together with the strong form of the perturbation Ampere equation (3a), i.e.,

$$
\begin{gathered}
\left(\partial_{t}\left(\mu_{p} \boldsymbol{h}\right), \boldsymbol{h}^{\prime}\right)_{\Omega_{p}}+\left(\partial_{t} \boldsymbol{b}_{s}, \boldsymbol{h}^{\prime}\right)_{\Omega_{c, p}}+\left(\sigma_{p}^{-1} \operatorname{curl} \boldsymbol{h}, \operatorname{curl} \boldsymbol{h}^{\prime}\right)_{\Omega_{c, p}} \\
-\left(\sigma_{p}^{-1} \boldsymbol{j}_{s}, \operatorname{curl} \boldsymbol{h}^{\prime}\right)_{\Omega_{c, p}}+<\boldsymbol{n} \times \boldsymbol{e}, \boldsymbol{h}^{\prime}>_{\Gamma_{e}}=0 \\
\forall \boldsymbol{h}^{\prime} \in F^{1}\left(\Omega_{p}\right)
\end{gathered}
$$

At the discrete level, the source quantities $\boldsymbol{j}_{S}$ and $\boldsymbol{b}_{S}$ initially given in the unperturbed mesh have to be expressed in the perturbed one. This can be done through a projection method (Geuzaine et al., 1999) with target quantities $\boldsymbol{j}_{S}$ and $\boldsymbol{b}_{s}$ defined in adequate function spaces. From (3a) and (4b), the projected $\boldsymbol{j}_{S}$ should have the same conformity as curl $\boldsymbol{h}$ (div-conform field), while from (3b) and (4a), the projected $\boldsymbol{b}_{s}$ should have the same conformity as $\boldsymbol{h}$ (curl-conform field). The field $\boldsymbol{b}_{s}(6)$ can be directly obtained via the projection of $\boldsymbol{h}_{u}$ from the unperturbed mesh to the perturbation one. However the field $\boldsymbol{j}_{s}(5)$ requires an intermediate step for obtaining $\boldsymbol{e}_{u}$. Both steps are developed in the next two subsections.

\section{Local projection of the unperturbed magnetic field}

The unperturbed magnetic field $\boldsymbol{h}_{u}$, first interpolated on the mesh of the unperturbed problem, can be projected (Geuzaine et al., 1999) on the one of the perturbation problem via the formulation

$$
\left(\boldsymbol{h}_{u, p r o j}, \boldsymbol{h}^{\prime}\right)_{\Omega_{c, p}}+\left(-\boldsymbol{h}_{u}, \boldsymbol{h}^{\prime}\right)_{\Omega_{c, p}}=0, \forall \boldsymbol{h}^{\prime} \in F^{1}\left(\Omega_{c, p}\right)
$$

The projected field $\boldsymbol{h}_{u, p r o j}$ has to be only known in the moving material region $\Omega_{c, p}$, which usually reduces the computational effort of the projection. Although the field $\boldsymbol{h}_{u, p r o j}$ can be defined as the gradient of a scalar potential, its direct projection is preferred to the one of this potential. Indeed the numerical derivative of such a projected scalar potential could give rise to numerical instabilities for the actual field of interest $\boldsymbol{h}_{u, p r o j}$.

When dealing with the perturbation problem, i.e. in $\Omega_{p}$, the projected field $\boldsymbol{h}_{u, p r o j}$ is renamed $\boldsymbol{h}_{u}$ for convenience.

\section{Electric problem in added moving conductive regions}

The source $\boldsymbol{j}_{s}$ in $\Omega_{c, p}$ is chosen to have the same conformity as curl $\boldsymbol{h}$, i.e. it is a div-conform field. From (5), (8b) and (8c), this can be satisfied through the definition of an electric vector potential $\boldsymbol{u}$ as primal unknown field, with

$$
\boldsymbol{d}_{u}=\operatorname{curl} \boldsymbol{u}
$$

satisfying (8b).

The electric model is governed by the weak form of (8a) in the sub-domain $\Omega_{c, p}$, i.e. an electric flux density 
conform formulation (Dular and Kuo-Peng, 2006),

$$
\begin{aligned}
& \left(\varepsilon_{u}^{-1} \operatorname{curl} \boldsymbol{u}, \operatorname{curl} \boldsymbol{u}^{\prime}\right)_{\Omega_{c, p}}+\left(\partial_{t}\left(\mu_{u} \boldsymbol{h}_{u}\right), \boldsymbol{u}^{\prime}\right)_{\Omega_{c, p}} \\
& +<\boldsymbol{n} \times \boldsymbol{e}_{u}, \boldsymbol{u}^{\prime}>_{\partial \Omega_{c, p}}=0, \forall \boldsymbol{u}^{\prime} \in F^{1}\left(\Omega_{c, p}\right),
\end{aligned}
$$

where the function space $F^{1}\left(\Omega_{c, p}\right)$ contains $\boldsymbol{u}$ and its associated test function $\boldsymbol{u}^{\prime}$ and has to be constrained with a gauge condition. At the discrete level, $\boldsymbol{u}$ is discretized with edge FEs and is associated a gauge condition by the tree co-tree technique.

The electric problem is here solved in the sub-region $\Omega_{c, p}$, whereas in (Dular and Kuo-Peng, 2006) it was globally solved in the whole non-conducting region. The trace of the electric field $\boldsymbol{e}_{u}$ (natural boundary condition in the surface integral term of (13)) is indeed known in some sense, not locally but through a surface integral via (9), which is enough. The expression of this surface integral for each test function $\boldsymbol{u}^{\prime}$ can be directly given by (9) written only for $\Omega_{p} \backslash \Omega_{c, p}$ (consequence of the similar conformity of $\boldsymbol{u}$ and $\boldsymbol{h}_{u}$ ), i.e.,

$$
\begin{gathered}
\left(\partial_{t}\left(\mu_{u} \boldsymbol{h}_{u}\right), \boldsymbol{u}^{\prime}\right)_{\Omega_{p} \backslash \Omega_{c, p}}+\left(\sigma_{u}^{-1} \operatorname{curl} \boldsymbol{h}_{u}, \operatorname{curl} \boldsymbol{u}^{\prime}\right)_{\Omega_{c} \backslash \Omega_{c, p}} \\
+<\boldsymbol{n} \times \boldsymbol{e}_{u}, \boldsymbol{u}^{\prime}>_{\partial \Omega_{c, p}}=0 .
\end{gathered}
$$

Adding (13) and (14), with normal vectors $\boldsymbol{n}$ exterior to $\Omega_{c, p}$ and $\Omega_{p} \backslash \Omega_{c, p}$ respectively, thus of opposite signs, the following equation is obtained

$$
\begin{gathered}
\left(\varepsilon_{u}^{-1} \operatorname{curl} \boldsymbol{u}, \operatorname{curl} \boldsymbol{u}^{\prime}\right)_{\Omega_{c, p}}+\left(\partial_{t}\left(\mu_{u} \boldsymbol{h}_{u}\right), \boldsymbol{u}^{\prime}\right)_{\Omega_{c, p}} \\
+\left(\partial_{t}\left(\mu_{u} \boldsymbol{h}_{u}\right), \boldsymbol{u}^{\prime}\right) \Omega_{\Omega_{p} \backslash \Omega_{c, p}}+\left(\sigma_{u}^{-1} \operatorname{curl} \boldsymbol{h}_{u}, \operatorname{curl} \boldsymbol{u}^{\prime}\right)_{\Omega_{c} \backslash \Omega_{c, p}}=0, \\
\forall \boldsymbol{u}^{\prime} \in F^{1}\left(\Omega_{c, p}\right) .
\end{gathered}
$$

Equation (15) illustrates well how $\boldsymbol{h}_{u}$ behaves as a source for determining $\boldsymbol{u}$ in $\Omega_{c, p}$ and $\partial \Omega_{c, p}$, both as a volume source $\partial_{\mathrm{t}}\left(\mu_{u} \boldsymbol{h}_{u}\right)$ by (8a) and a boundary source naturally converted to a volume source by (14) (via a volume integration limited to the layer of FEs touching $\partial \Omega_{c, p}$ in $\left.\Omega_{p} \backslash \Omega_{c, p}\right)$.

As a result, using (5), $\sigma_{u}=0,(8 c),(12)$ and the solution $\boldsymbol{u}$ of (15), the source quantity $\boldsymbol{j}_{s}$ is obtained with the desired conformity, i.e.,

$$
\boldsymbol{j}_{S}=\sigma_{p} \boldsymbol{e}_{u}=\sigma_{p} \varepsilon_{u}^{-1} \boldsymbol{d}_{u}=\sigma_{p} \varepsilon_{u}^{-1} \operatorname{curl} \boldsymbol{u}
$$

\section{E. Back to the sequence of perturbation problems}

The source quantities $\boldsymbol{j}_{s}(5)$, particularized with the expression (16), and $\boldsymbol{b}_{S}(6)$ can then be used in (10), the solution of which gives the eddy current density in $\Omega_{c, p}$ and the field distortions.

Once the unperturbed or source solution has been calculated, any new position of the moving region $\Omega_{c, p}$ asks for (a) projecting $\boldsymbol{h}_{u}$ in its own mesh, (b) directly expressing the associated source $\boldsymbol{b}_{S}$, (c) determining the source $\boldsymbol{j}_{s}$ via an electric problem solution, and eventually (d) computing the field distortion introduced by the moving region.

The time derivatives involved in both the magnetodynamic perturbation formulation (10) and the electric formulation (15) are simply expressed in the fixed frame of the moving region. Given that the mesh of this region remains the same for any position, direct expressions (in terms of differences of time values of nodal and edge quantities) can be written.

Similarly to what was demonstrated in (Sabariego and Dular, 2006; Dular and Sabariego, 2007), the use of the sources (5) and (6), by integrating some contributions only in $\Omega_{c, p}$, constitutes an efficient way to calculate the impedance changes of the coils of the unperturbed problem. This is of interest e.g. in eddy-current nondestructive testing applications.

\section{Application}

A transverse flux system (Fig. 2) is considered as a 2$\mathrm{D}$ test problem to illustrate and validate the whole perturbation procedure applied to the movement modeling (two coils with 1000 turns, direct current $2.5 \mathrm{~A}$, two cores of relative permeability $\mu_{r \text {, core }}=100$ ). The moving region $\Omega_{c, p}$ is a conductive copper piece $\left(\sigma_{\text {piece }}=5.910^{7} \Omega^{-1} \mathrm{~m}^{-1}, \mu_{r \text {, piece }}=1\right.$, exterior size $75 \mathrm{~mm}$ $\times 200 \mathrm{~mm}$, width at mid-height $25 \mathrm{~mm}$ ).

Fig. 2 shows examples of meshes for the unperturbed and perturbation problems. Figs. 3-6 illustrate the sequence of associated solutions to be considered with the developed perturbation method (for different positions and a speed of $28 \mathrm{~m} / \mathrm{s}$ ), i.e., the unperturbed field $\boldsymbol{h}_{u}$ (Fig. 3), the electric vector potential $\boldsymbol{u}$ (Fig. 4), the unperturbed electric field $\boldsymbol{e}_{u}$, the perturbation current density $\boldsymbol{j}$ (Fig. 5) and the perturbation magnetic flux density $\boldsymbol{b}$ (Fig. 6). The local perturbation fields have been verified to be very similar to those obtained with the classical FE technique. For the chosen speed, skin effects on the eddy current density in the moving piece can be observed (Fig. 5, right), although the source electric field is quite uniform along the piece width (Fig. 5, left).

The Joule losses versus the position of the moving piece are depicted in Fig. 7 for different extensions of the perturbation domain. The reference solution is calculated from the conventional FE technique, the major drawbacks of which are the need of remeshing the whole system and a new complete FE solution for each new position of the moving piece. For a movement in a well defined direction, the moving band technique could be applied as well. Note that the proposed technique is not constrained by the direction of the movement, it naturally and easily enables free movement modeling. The interest of extending the studied perturbation domain $\Omega_{p}$ up to infinity, through a transformation technique (Imhoff $e t$ 
al., 1990), is clearly pointed out, which justifies the preference for perturbation boundary conditions at infinity. The Joule losses in the moving piece are also shown in Fig. 8 for different speeds, proving their increase with the speed.

For the considered problem, a sequence of 40 perturbation solutions, covering the same number of time steps for the movement modeling, has been solved with a speed-up factor of 60 in comparison to the conventional FE technique.

\section{Conclusion}

A sub-problem approach has been developed for efficiently solving magnetodynamic problems in moving systems. It first asks for solving a problem without any moving parts, which then gives sources for these parts considered alone in their own frames, with their associated fixed meshes, for successive positions during the time evolution.

Each problem uses an adapted mesh, which necessitates the projection of fields between meshes. The advantages of defining volume sources limited to the moving regions have been highlighted. These mainly concern the boundary conditions possibly defined at infinity, the reduced computational efforts for projecting and evaluating the sources, as well as the calculation of impedance variations. The sub-problem approach also provides a simple method to express the time derivatives in moving frames.

As a consequence, a significant speed-up of analyses of moving systems is obtained. Further developments concern the force computation on the moving regions as well as an additional iterative process for strongly coupled sub-problems and nonlinear analyses.

\section{REFERENCES}

Badics, Z. et al. (1997), "An effective 3-D finite element scheme for computing electromagnetic field distorsions due to defects in eddycurrent nondestructive evaluation", IEEE Trans. Magn., Vol. 33, No. 2, pp. 1012-1020.

Dular, P. and Kuo-Peng, P. (2006), "Dual finite element formulations for the three-dimensional modeling of both inductive and capacitive effects in massive inductors", IEEE Trans. Magn., Vol. 42, No. 4, pp. 743-746.

Dular, P., Kuo-Peng, P., Geuzaine, C., Sadowski, N and Bastos, J.P. A. (2000), "Dual magnetodynamic formulations and their source fields associated with massive and stranded inductors", IEEE Trans. Magn., Vol.36, No.4, pp.3078-3081.

Dular, P. and Sabariego, R.V. (2007), "A Perturbation Method for Computing Field Distortions due to Conductive Regions with hConform Magnetodynamic Finite Element Formulations", IEEE Trans. Magn., Vol. 43, No. 4, in press.

Geuzaine, C., Meys, B., Henrotte, F., Dular, P. and Legros, W. (1999), "A Galerkin projection method for mixed finite elements", IEEE Trans. Magn., Vol. 35, No.3, pp. 1438-1441.

Imhoff, J.F., Meunier, G., Brunotte, X. and Sabonnadière, J.C. (1990), "An original solution for unbounded electromagnetic 2D and 3D problems throughout the finite element method", IEEE Trans. Magn., Vol. 26, No. 5, pp. 1629-1631.

Sabariego, R. V. and Dular, P. (2006), "A perturbation technique for the finite element modelling of nondestructive eddy current testing", in Electromagnetic Fields in Mechatronics, Electrical and Electronic Engineering, ser. Studies in Applied Electromagnetics and Mechanics, A. Krawczyk, S. Wiak and X.M. Lopez-Fernandez, Eds., Amsterdam, The Netherlands, IOS Press, vol. 27.
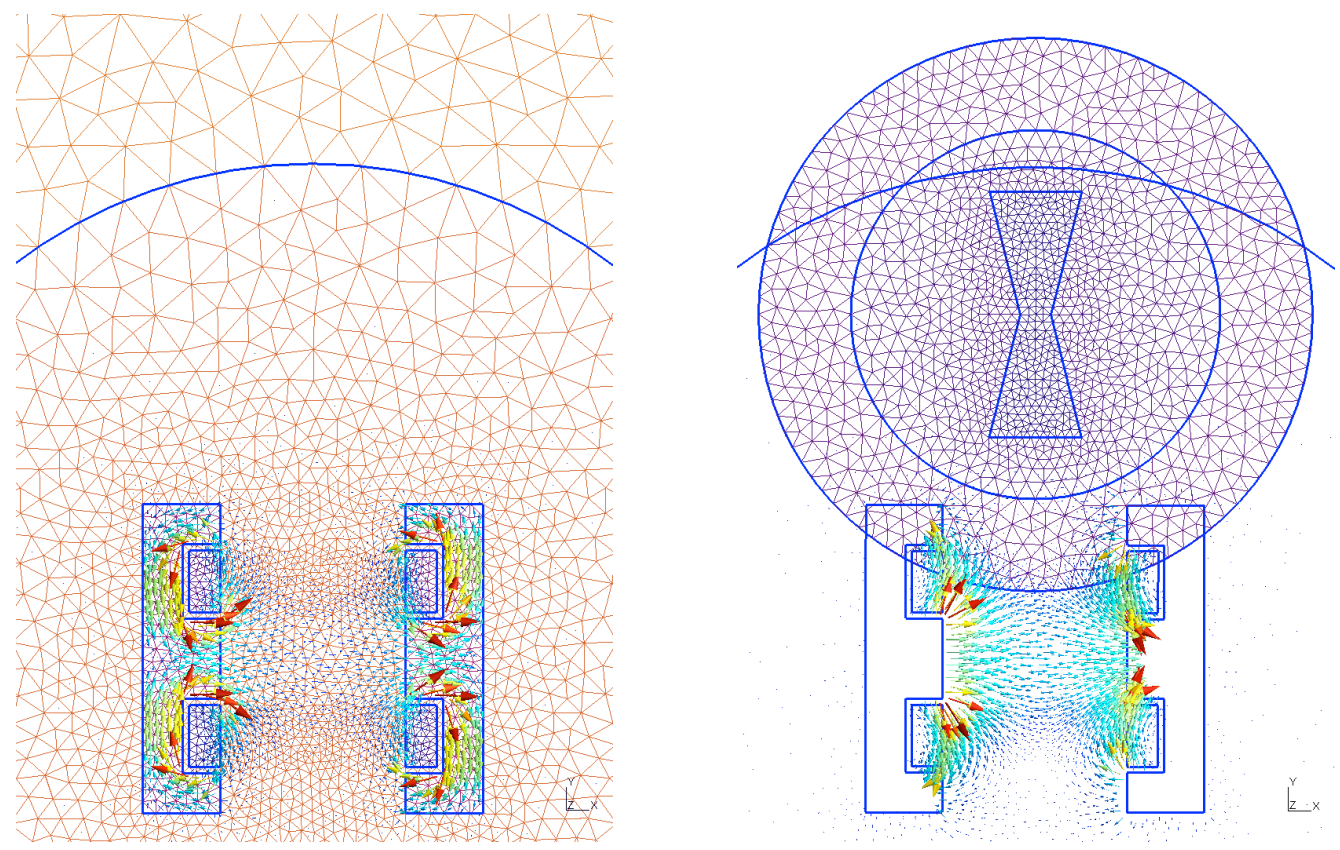

Fig. 2. Meshes of the unperturbed problem (left; with field $\boldsymbol{b}_{u}$ ) and perturbation problem with infinite boundaries around the moving piece (right; (Imhoff et al., 1990); with field $\boldsymbol{h}_{u}$ ). Any intersection of perturbation boundaries (or $\Omega_{p} \backslash \Omega_{c, p}$ ) with the unperturbed problem material regions is allowed. 

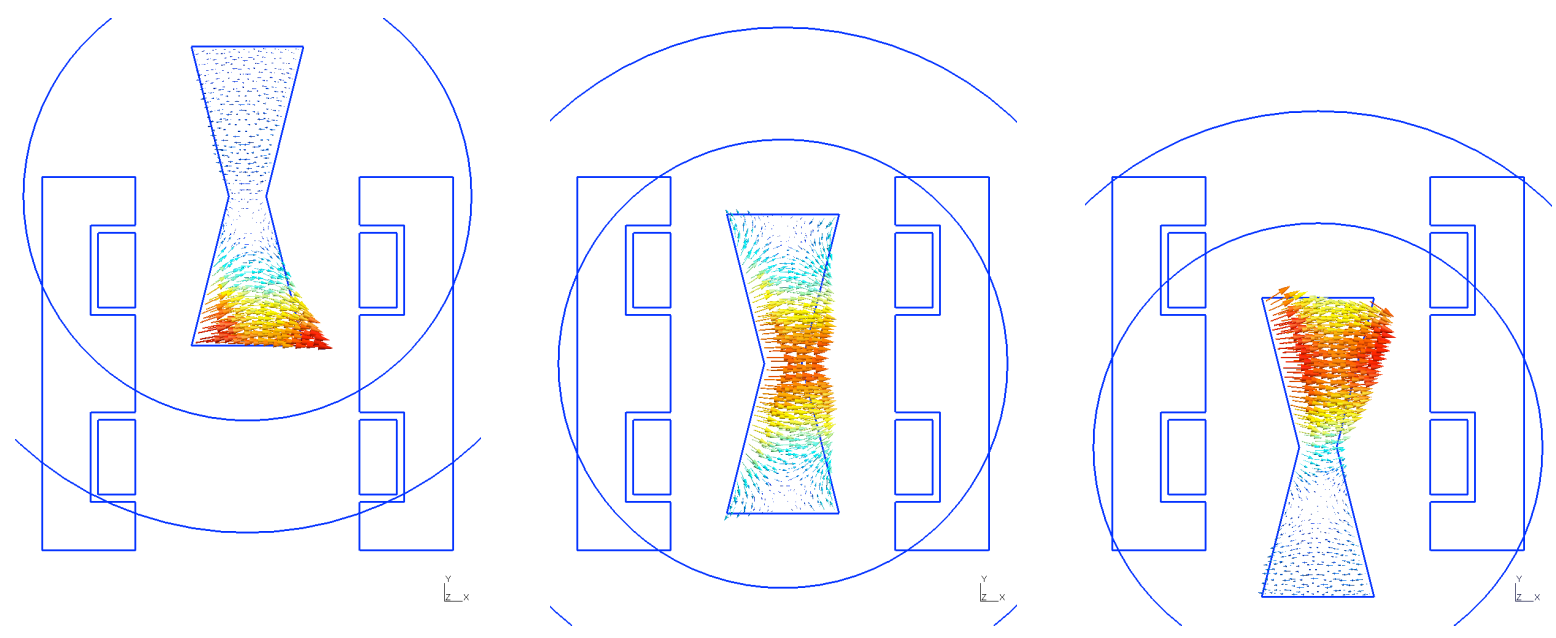

Fig. 3. The unperturbed field $\boldsymbol{h}_{u}$ projected in $\Omega_{c, p}$ for different positions of the moving piece (positions $16.8 \mathrm{~mm}, 28 \mathrm{~mm}$ and $33.6 \mathrm{~mm}$ ).
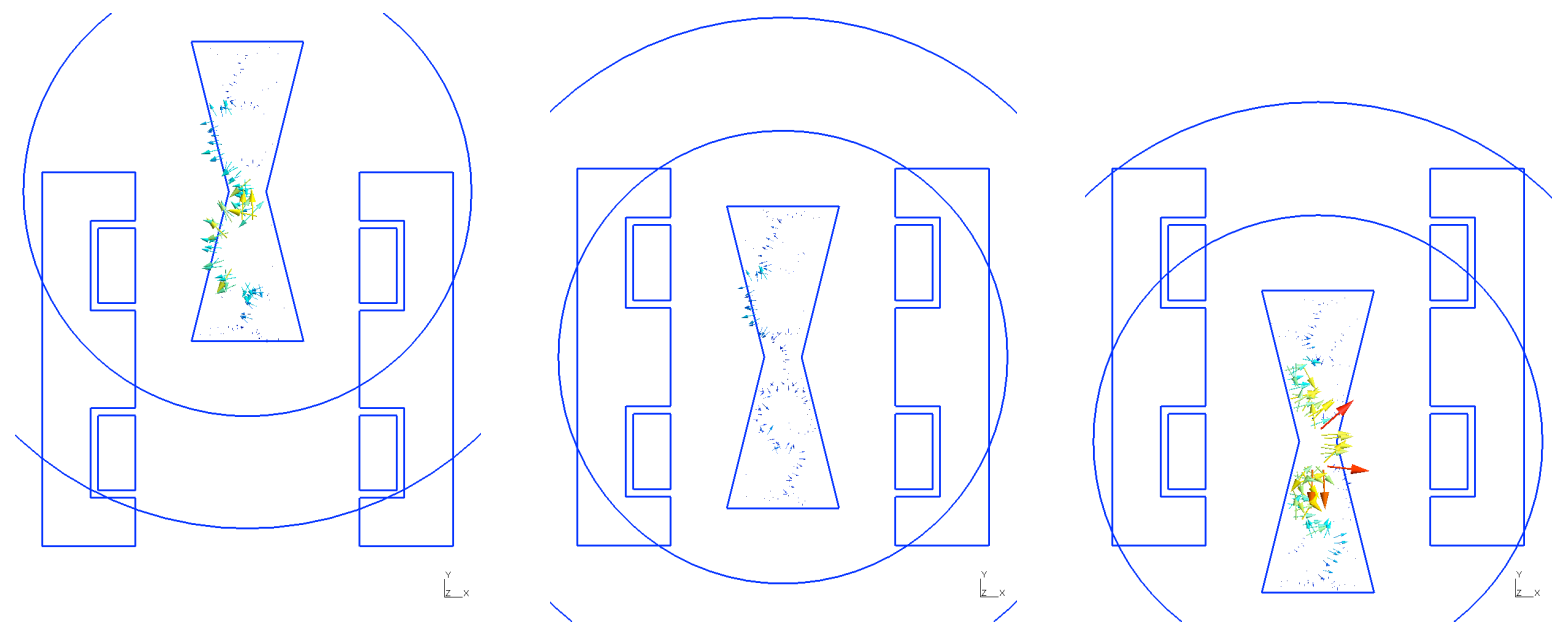

Fig. 4. The electric vector potential $\boldsymbol{u}$ in $\Omega_{c, p}$, gauged by the tree co-tree technique and the source of which is $\boldsymbol{h}_{u}$ (for different positions of the moving piece).
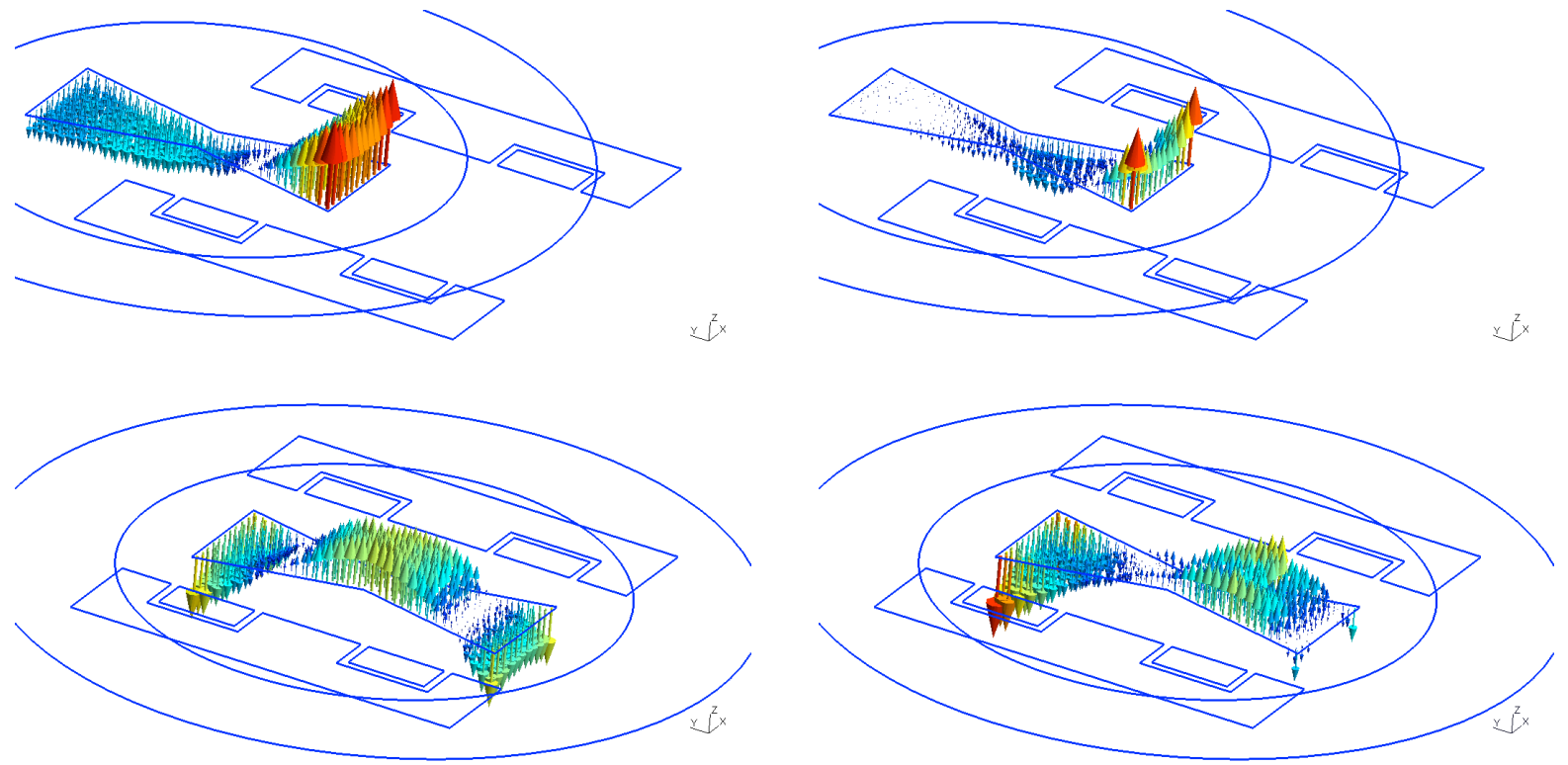

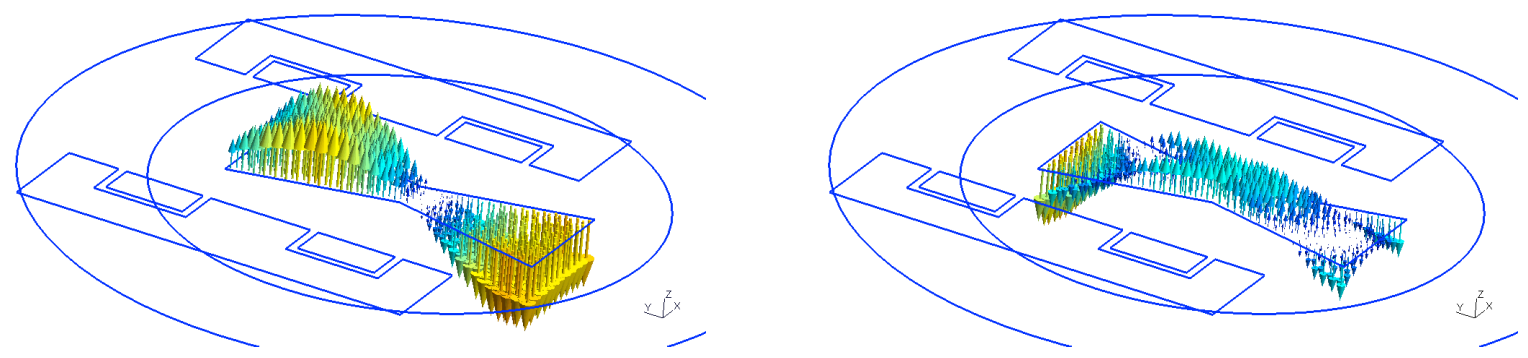

Fig. 5. The ensuing unperturbed electric field $\boldsymbol{e}_{u}=\varepsilon_{u}{ }^{-1} \operatorname{curl} \boldsymbol{u}$ (left) and the perturbation current density $\boldsymbol{j}=\operatorname{curl} \boldsymbol{h}$ in $\Omega_{c, p}($ right); for different positions of the moving piece.
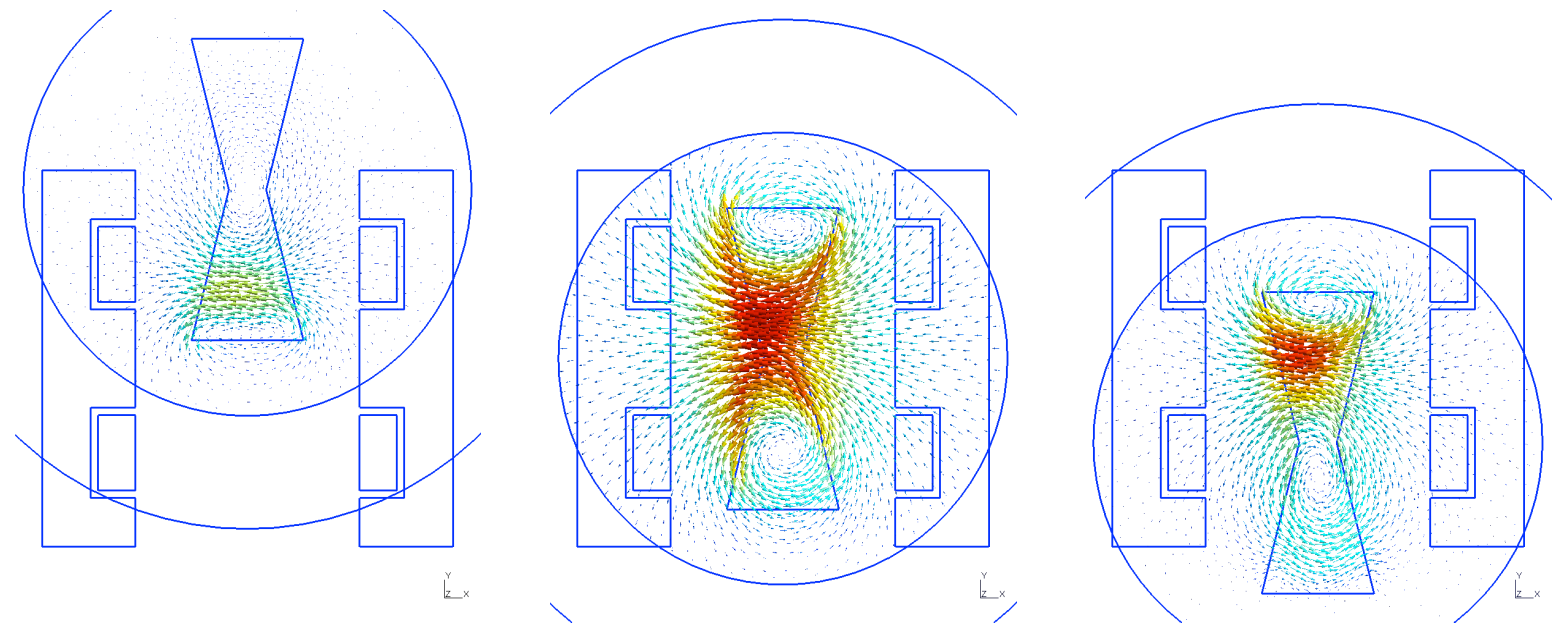

Fig. 6. The perturbation magnetic flux density $\boldsymbol{b}=\boldsymbol{\mu} \boldsymbol{h}$ in $\Omega_{p}$; for different positions of the moving piece.

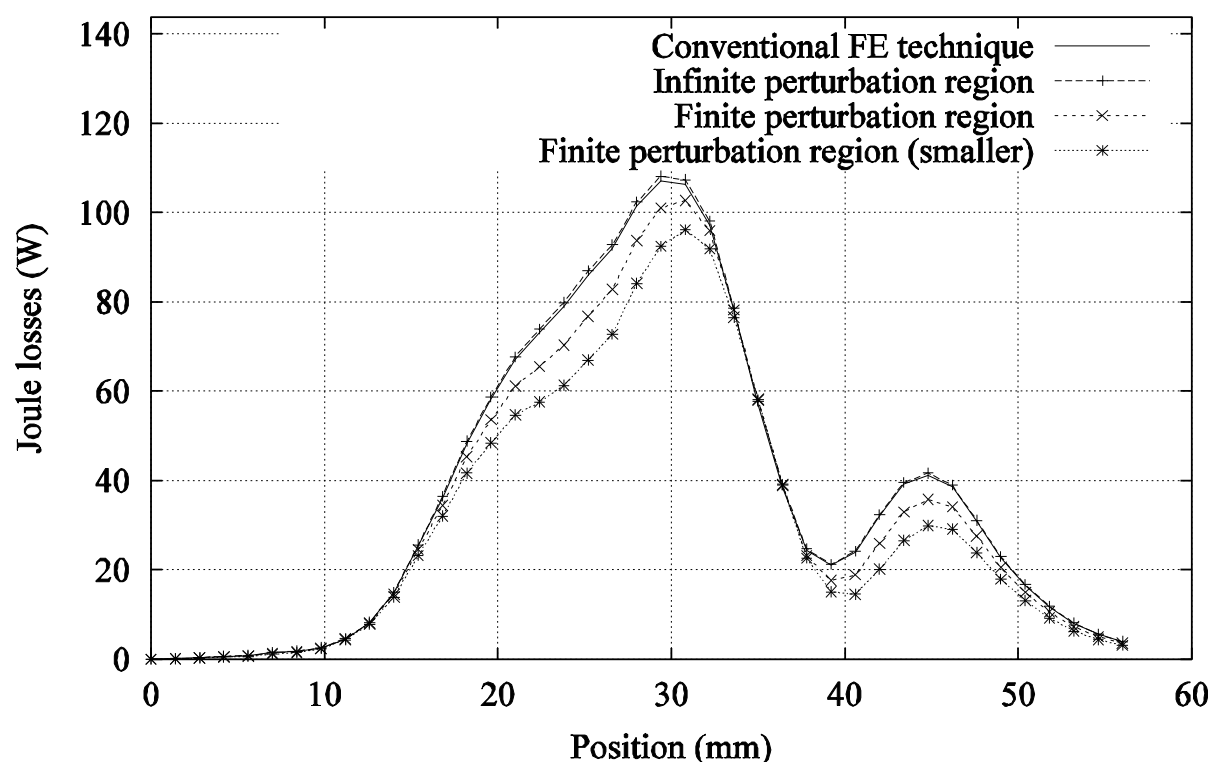

Fig. 7. Joule losses versus the position of the moving region (speed $28 \mathrm{~m} / \mathrm{s}$ ) for different sizes of the perturbation region $\Omega_{p}$. 


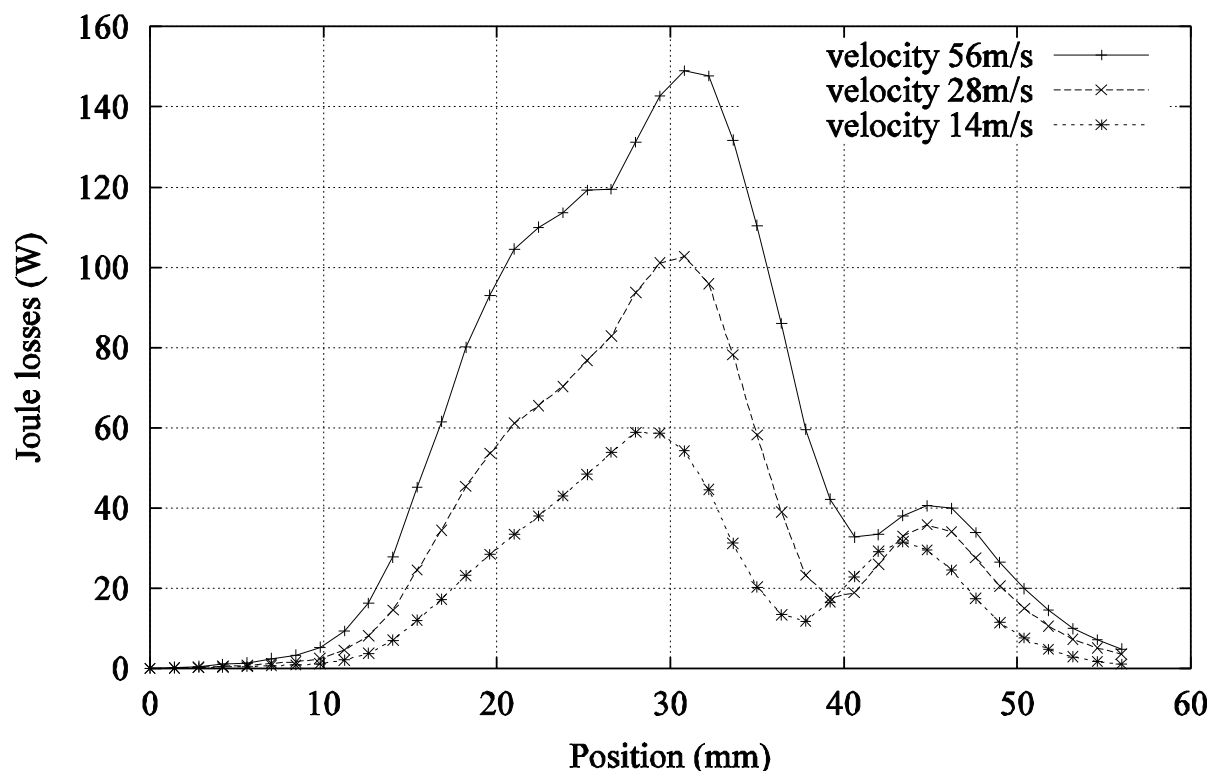

Fig. 8. Joule losses versus the position of the moving region for different speeds (with infinite perturbation region). 